\title{
MEASUREMENT OF SEXTUPOLE ORBIT OFFSETS IN THE APS
} STORAGE RING *

\section{Abstract}

Horizontal orbit errors at the sextupoles in the Advanced Photon Source (APS) storage ring can cause changes in tune and modulation of the beta functions around the ring. To determine the significance of these effects requires knowing the orbit relative to the magnetic center of the sextupoles. The method considered here to determine the horizontal beam position in a given sextupole is to measure the tune shift caused by a change in the sextupole strength. The tune shift and a beta function for the same plane uniquely determine the horizontal beam position in the sextupole. The beta function at the sextupole was determined by propagating the beta functions measured at nearby quadrupoles to the sextupole location. This method was used to measure the sextupole magnetic center offset relative to an adjacent beam position monitor (BPM) at a number of sextupole locations. We report on the successes and problems of the method as well as an improved method.

\section{INTRODUCTION}

Given the strong sextupoles present in third-generation light sources, miscentering of the beam in the sextupoles can seriously impact one's ability to model the machine's behavior. This affects one's ability to correct the orbit, adjust the tunes, and perform other corrections that tend to make use of data from modeling. It may also have an adverse effect on dynamic aperture and injection.

There are several possible sources of such miscentering. First, a sextupole may simply be improperly aligned. Second, an unknown or mistaken value for a BPM offset may result in steering off axis in the sextupole. Third, the beam may be moved deliberately to steer for a user. (At APS, final beam alignment for users is performed by steering of the electron beam.) Fourth, since some sextupoles are in dispersion areas, a systematic miscentering may result from a particular choice of the if frequency.

At APS, many BPM offsets are derived using a scanning technique using a quadrupole and a corrector bump [1]. This permits finding the offsets relative to quadrupoles for those BPMs that are adjacent to quadrupoles. The method relies on the fact that if the beam is centered in a quadrupole, then changing the strength of that quadrupole does not change the orbit. Because this method is relatively straightforward to implement, we used it as the definition of our BPM offsets. The assumption was that the sextupoles were well-aligned relative to the quadrupoles, so that steering to the center of quadrupoles would also center the beam in the sextupoles.

\footnotetext{
* Work supported by U.S. Department of Energy, Office of Basic Energy Sciences, under Contract No. W-31-109-ENG-38.
}

\begin{abstract}
However, we encountered persistent disagreen $190 \mathrm{~g}$ between our model of the ring and mealeg nto of the beta functions. Hence, a program to measure tho befm position in sextupoles directly was undertaken. Note that while we sometimes speak of measuring sextupole "offsets" or "positions" and of "sextupole miscentering," we are in fact measuring the position of the beam relative to the sextupole center for a particular lattice configuration and steering.
\end{abstract}

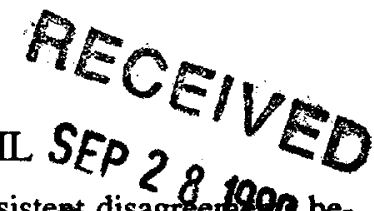

\section{PRINCIPLE OF THE MEASURMENT}

The measurement relies on the quadrupole field component generated by a displaced sextupole magnet. It also makes use of the existence of individual power supplies for the 280 sextupoles and 400 quadrupoles in the APS. The effective geometric focusing strength $\left(K_{1}\right)$ seen by a beam displaced by $x$ from the magnetic center of a sextupole of geometric strength $K_{2}$ is just $K_{2} x$. If the sextupole strength is changed between states 1 and 2 with no change in orbit, then the tune change is related to the change in $K_{2}$ by a well-known [2] equation, giving

$$
\Delta \nu= \pm \frac{\beta \Delta K_{2} x L_{s}}{4 \pi},
$$

where $L_{s}$ is the length of the sextupole, the $+(-)$ sign is used if horizontal (vertical) tune data is used, and the $\beta$ function should be for the same plane as the tune change. In order to determine $x$, we change the strength of the sextupole and measures the change in tune. To make the tune change as large as possible, we chose to change the sextupole from zero to maximum current. The value of $\Delta K_{2} L_{s}$ is then $4.974 \mathrm{~m}^{-2}$ [3] for APS sextupoles.

In order to eliminate spurious tune changes due to orbit motion elsewhere in the ring caused by the change in the sextupole field, we employed continuous orbit correction and a settling period (30-60s) to allow correction of any orbit perturbation. Typical perturbations were 20-30 $\mu \mathrm{m}$ peak and were easily corrected.

The beta function value needed to compute $x$ was originally taken from the model. Later, we implemented a refinement of the technique that involves using measured beta functions from two quadrupoles that bracket the sextupole.

\section{MEASUREMENT TECHNIQUE AND DATA ANALYSIS}

The principle of this measurement is clearly quite simple, and it was readily implemented using existing software tools, notably the SDDS (Self-Describing Data Sets) toolkit $[1,4,5]$. The measurement is available via a GUI interface built using the Tcl/Tk script language. The script uses SDDS tools for data collection, analysis, and display. 


\section{DISCLAIMER}

This report was prepared as an account of work sponsored by an agency of the United States Government. Neither the United States Government nor any agency thereof, nor any of their employees, make any warranty, express or implied, or assumes any legal liability or responsibility for the accuracy, completeness, or usefulness of any information, apparatus, product, or process disclosed, or represents that its use would not infringe privately owned rights. Reference herein to any specific commercial product, process, or service by trade name, trademark, manufacturer, or otherwise does not necessarily constitute or imply its endorsement, recommendation, or favoring by the United States Government or any agency thereof. The views and opinions of authors expressed herein do not necessarily state or reflect those of the United States Government or any agency thereof. 


\section{DISCLAIMER}

Portions of this document may be illegible in electronic image products. Images are produced from the best available original document. 
The tune measurements were taken with a HewlettPackard Vector Signal Analyzer (HP VSA), which has low noise and fast averaging compared to a typical network analyzer, using a frequency chirp to drive the beam. To save time and allow higher tune measurement resolution, we narrowed the span of the analyzer to include only one of the tunes. We chose to use the vertical tune for most measurements as this increases $\Delta \nu$, given that we have $\beta_{y}>\beta_{x}$ at most of the sextupoles of interest. Further, the vertical tune is less subject to drift and wobble than the horizontal tune.

The script reads the tune spectra from the HP VSA for the two sextupole settings ( 0 and full current). Typical spectra are shown in Figure 1. The script processes the tune spectra using either a smoothing and peakfinding algorithm or a correlation-based algorithm. The results are very close for the two algorithms, with maximum differences being equivalent to about $30 \mu \mathrm{m}$ in sextupole position.

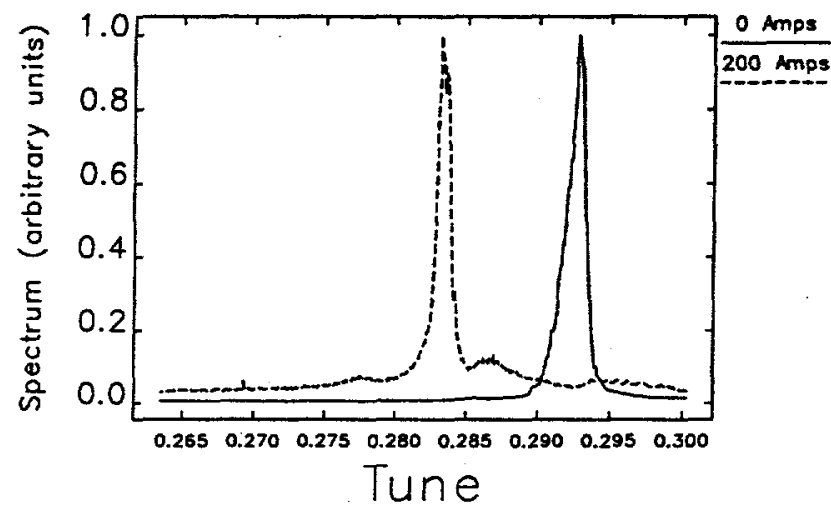

Figure 1: Typical spectra from a measurement of beam position in a sextupole

The same instrument and software tools are used for the beta function measurements. Indeed, the equation underlying the beta function measurement is simply a variant of Eq. (1), namely $\beta=\Delta K_{1} \beta L_{S} /(4 \pi)$. We have verified using simulations that this equation is accurate when used for a single quadrupole at a time or for groups of up to 40 quadrupoles; that is, any perturbation of the lattice due to the measurement is negligible. The beta function measurement script takes five tune measurements for five different values of the quadrupole strength. The beta function is computed from the slope of the tune vs quadrupole strength, where the strength itself is deduced from the excitation curve of the magnet. The script restores the tunes by iteratively adjusting the quadrupole current, so that the lattice is not perturbed by successive measurements. This means we do not have to rely on knowing the hysteresis behavior of the magnet in order to restore the lattice.

Figure 2 shows a histogram of the measurements made to date, for $\mathbf{2}$ and $S 3$ sextupoles, that bracket the dipoles in our double bend acromat (DBA) lattice. The mean position is $-0.25 \pm 0.04 \mathrm{~mm}$. For the S2 sextupoles (which are in a nominally zero-dispersion location), the mean is $-0.13 \pm$ $0.05 \mathrm{~mm}$. For the $\mathrm{S} 3$ sextupoles (in a dispersion location), the mean is $-0.37 \pm 0.05 \mathrm{~mm}$. This suggests that we are systematically off-center in the sextupoles largely due to the particular value of if frequency we use.

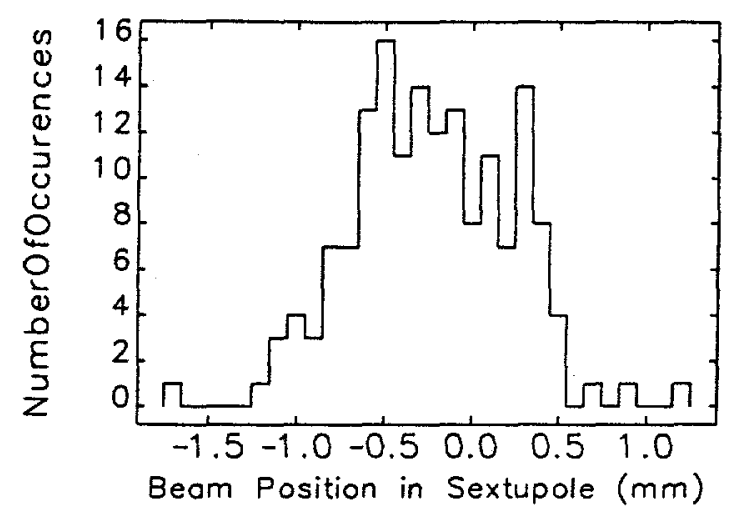

Figure 2: Histogram of measured beam positions in sextupoles

As mentioned in the introduction, one reason for wanting to know the beam position in the sextupoles is to evaluate the effect on the lattice. Although we have only made measurements for 160 of the 280 sextupoles, it is interesting to compute the beta function resulting from the beam offsets in these sextupoles. This is shown in Figure 3. In practice, such beats are corrected using a singular value decomposition (SVD) technique [6] that does not require knowing the beam position in the sextupoles. However, application of that technique does not provide an explanation of the presence of beta beats. Although our data is incomplete and cannot be taken to represent the actual beta function modulation, it does demonstrate the possibility that any such modulations may be due to the position of the beam in the sextupoles.

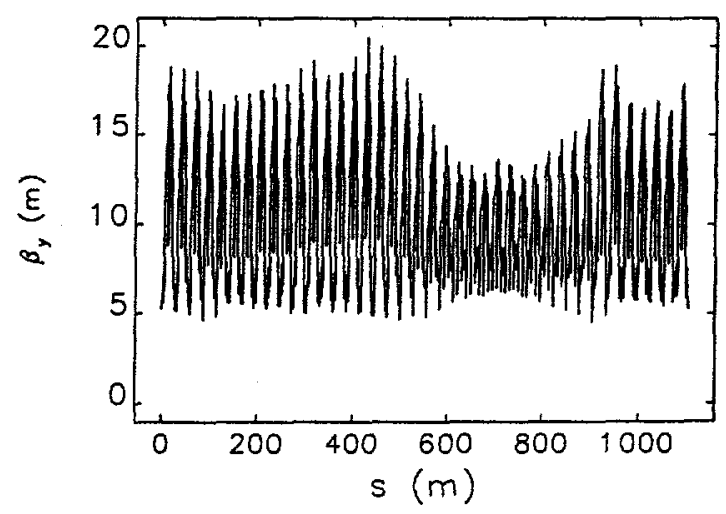

Figure 3: Computed vertical beta function for the APS due to beam positions in the 160 sextupoles for which measurements were done. APS has 280 sextupoles plus other potential sources of focusing errors, so this figure does not purport to represent the actual beta function.

\section{TESTS OF THE METHOD}

We tested the method in two ways: First, we checked the reproducibility of the measurement; when repeated within 
a short time, this was very good, with successive measurements reproducing at the $2-\mu \mathrm{m}$ level imposed by our tune measurement resolution. Second, we checked the linearity and slope by making successive measurements with the beam deliberately steered to different positions in a sextupole. This was done for two sextupoles. The linearity was generally good, but the data showed slope errors; i.e., the change in BPM reading over the change in position deduced from the sextupole-based measurement was not unity. For example, in one case we found a reproducible $30 \%$ error in the slope, as seen in Figure 4. In another case we found a $50 \%$ error. Possible sources of this error are the various calibrations of the BPM, sextupole, and quadrupole, plus the particular bump shape that was used.

There is evidence from response matrix measurements of $15-25 \%$ errors in the calibration of the two BPMs in question. Since this is based on comparing a measured matrix [7] to a computed matrix using a model that matches the average beta functions [8], it is not necessarily accurate for a given sector. However, because $30-50 \%$ of magnet calibration errors are difficult to conceive, we believe that BPM calibration accounts for most of the discrepancy. If this is correct, then the beam position measurements in the sextupoles are reliable. These measurements do not rely on BPMs, but only on tune measurement.

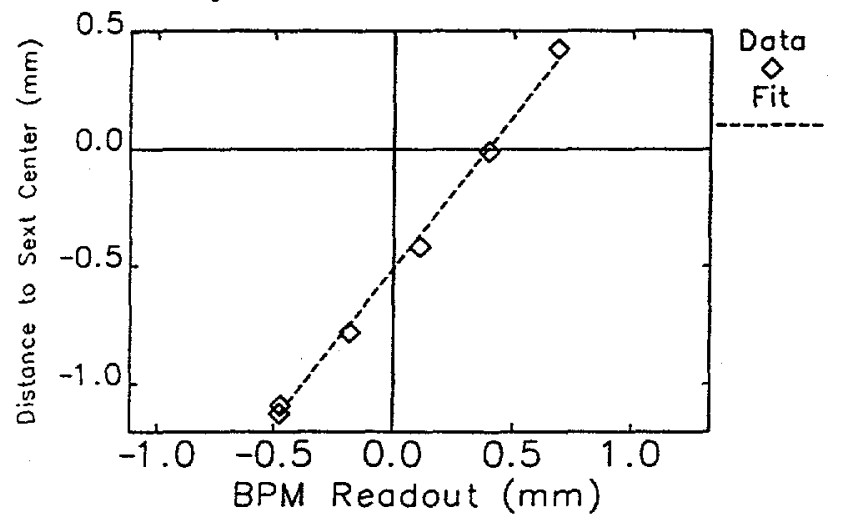

Figure 4: Linearity test of measurement of beam position in a sextupole

Even if the position measurements are accurate, the discrepancy makes it impossible to use the data to center the beam in the sextupoles. Because of this, we have developed a different technique that obviates the need to know the magnet calibrations. Specifically, we will use beam bumps in the sextupoles to steer the beam until the tune change due to changing a sextupole current is nulled out. The readout of the nearest BPM tells us where to steer the beam in order to center it in the sextupole. As long as the BPM calibration is not changed, this data could be used for beam centering even if the calibration is not good in absolute terms. This method also requires no measurement of beta functions and, being iterative, it does not rely on the linearity of the BPMs. An initial test of this method showed that it converged to the accuracy of our tune measurements after three to four iterations.
One problem discovered when doing the linearity tests was that the results depended on the type of beam bump used (i.e., the location and coefficients of the corrector magnets). We interpret this to mean that different types of local bumps may have sufficiently different shapes that the relationship between the position at the nearest BPM and the position in the sextupole changes appreciably. Hence, in choosing the beam bump to use for the new method, care needs to be taken that the beam is moved in a parallel fashion through the sextupole and nearby BPMs. This also implies that we may have a practical difficulty in steering precisely to the center of all sextupoles, as such bumps are likely to move the beam in several sextupoles.

\section{CONCLUSION}

A method of measuring the beam position in sextupoles by measuring the tune shift caused by a change in sextupole strength has been presented. The method is shown to be linear with respect to BPM readout, although the slope was in error by $30-50 \%$. This implies that our measured positions may have errors of this magnitude; however, we believe much of the error in the two cases studied is attributable to BPM calibration and hence that the measured positions are accurate. The error in each individual measurement was found to reproduce at the $2-\mu \mathrm{m}$ level (the limit imposed by the tune measurements) when measurements were taken in quick succession. A modified technique that directly finds the beam-centering value to which to steer on a nearby BPM was also discussed.

\section{REFERENCES}

[1] M. Borland, L. Emery, N. Sereno, "Doing Accelerator Physics Using SDDS, UNIX, and EPICS," Proc. 1995 ICALEPCS, Oct. 30 - Nov. 3, 1995, Chicago, Illinois, pp. 382-391 (1996).

[2] M. Sands, "The Physics of Electron Storage Rings: An Introduction," SLAC-121, Stanford Linear Accelerator Center, May 1979.

[3] C. Doose, ANL/APS, private communication.

[4] M. Borland, "A Self-Describing File Protocol for Simulation Integration and Shared Postprocessors," Proc. 1995 PAC, May 1-5, 1995, Dallas, Texas, pp. 2184-2186 (1996).

[5] M. Borland, "A Universal Postprocessing Toolkit for Accelerator Simulation and Data Analysis," Proc. 1998 ICAP Conference, Sept. 14-18, 1998, Monterey, California, to be published.

[6] L. Emery, "Dispersion and Betatron Function Correction in the APS Storage Ring Using Singular Value Decomposition," these proceedings.

[7] J. Carwardine, ANLAPS, private communication.

[8] L. Emery, ANL/APS, private communication. 